MINI-SYMPOSIUM

\title{
Free radical production by dysfunctional eNOS
}

\author{
M C Verhaar, P E Westerweel, A J van Zonneveld, T J Rabelink
}

Heart 2004;90:494-495. doi: 10.1136/hrt.2003.029405

E ndothelium derived nitric oxide (NO) plays a major role in cardiovascular homeostasis. It has important antiatherosclerotic properties which include regulation of vasomotor tone and vessel wall permeability, suppression of leucocyte adhesion to the endothelial surface, inhibition of platelet aggregation, and inhibition of vascular smooth muscle cell migration and proliferation. The important role of NO in the cardiovascular system is highlighted by key observations in animal models. Inhibition of endothelial NO synthase (eNOS), the enzyme that catalyses endothelial NO synthesis, accelerates atherogenesis. Similarly, genetic deletion of eNOS in mice leads to hypertension, defective vascular remodelling, vascular thrombosis, and enhanced leucocyteendothelial cell interactions. In humans, all major cardiovascular risk factors, including hypercholesterolaemia, hypertension, diabetes, and smoking, have been associated with endothelial dysfunction, characterised by impaired NO bioavailability. Importantly, the impairment of NO mediated endothelial function is an independent predictor of adverse cardiac events. ${ }^{1}$ Taken together, current data strongly suggest that impaired NO activity is a crucial factor in the pathogenesis of cardiovascular disease. Improving endothelial NO bioavailability in vivo may reduce cardiovascular risk and has emerged as a major therapeutic goal.

\section{SYNTHESIS AND DEGRADATION OF NITRIC OXIDE}

In vivo NO bioactivity is determined by the balance between synthesis and degradation of NO. The biosynthesis of endothelial NO is catalysed by the enzyme eNOS and requires the amino acid L-arginine, nicotinamide adenine dinucleotide phosphate (NADPH), and molecular oxygen as substrates, as well as several cofactors and prosthetic groups. Inactivation of NO may occur by its reaction with oxyhaemoglobin in erythrocytes but also by reacting with superoxide anions, resulting in the formation of peroxynitrite. Increased superoxide production is a feature of various vascular disease states and is now recognised as an important determinant of impaired NO activity in vivo. ${ }^{2}$ Potential sources of superoxide in the vessel wall include mitochondria, cytochrome P450type enzymes, cyclooxygenase, lipoxygenase, NAD(P)H oxidases, and xanthine oxidase. Interestingly, in vitro studies in purified eNOS have shown that eNOS itself, beside its key role in NO production, may be a potential source of superoxide. ${ }^{3}$ Accordingly, in animal models of atherosclerosis removal of the endothelial monolayer or infusion of a selective NOS antagonist not only prevented NO formation but also inhibited increased formation of superoxide. Recent reports, demonstrating that diet induced fatty streak formation was paradoxically reduced in mice lacking eNOS $^{4}$ whereas chronic overexpression of eNOS accelerated atherosclerotic lesion formation in apoE-deficient mice, ${ }^{5}$ provide in vivo support for such a dual role of eNOS and identify eNOS (dys)function rather than eNOS expression as a key target for anti-atherosclerotic therapies.

\section{ENDOTHELIAL NOS}

Endothelial NOS is a dimeric, bidomain enzyme consisting of a C-terminal reductase domain which binds NADPH, flavin mononucleotide (FMN), and flavin adenine dinucleotide (FAD), an N-terminal oxidase domain which binds a prosthetic heme group, tetrahydrobiopterin (BH4), oxygen, and L-arginine and a regulatory calmodulin binding sequence. Under physiological conditions, after binding of $\mathrm{Ca}^{2+} /$ calmodulin between the oxygenase and reductase domain, electrons are donated by reduced NADPH and shuttled through the reduced flavins toward the oxidase domain. At the heme site molecular oxygen is reduced and incorporated into L-arginine to form $\mathrm{NO}$ and L-citrulline. The essential cofactor BH4 has been shown to be a key factor in eNOS catalysis. Experiments in purified eNOS showed that in the absence of BH4, "eNOS uncoupling" may occur-that is, uncoupling of NADPH oxidation and NO synthesis, with oxygen instead of L-arginine as terminal electron acceptor, resulting in the formation of superoxide. ${ }^{6}$ The crucial role of $\mathrm{BH} 4$ in eNOS coupling was supported by studies in both endothelial cells as well as isolated vessels showing that reduction of intracellular $\mathrm{BH} 4$ concentrations by inhibition of GTP cyclohydrolase I, the rate limiting enzyme for BH4 synthesis, resulted in a reduction of NO synthesis and enhanced superoxide generation, which could be reversed by incubation with sepiapterin, substrate for BH4 synthesis. ${ }^{7}$ Importantly, several clinical studies have demonstrated beneficial effects of $\mathrm{BH} 4$ administration on endothelial function in patients with cardiovascular risk factors, such as hypercholesterolaemia, smoking, hypertension, and diabetes or coronary artery disease. ${ }^{8} 9$ Thus, in patients at increased cardiovascular risk, the decreased availability of $\mathrm{BH} 4$ in the vessel wall with subsequent eNOS dysfunction may be a crucial determinant of impaired NO mediated endothelial function.

\section{BH4 BIOAVAILABILITY}

The underlying reason for the decreased $\mathrm{BH} 4$ bioavailability in endothelial dysfunction has not been fully elucidated but may be related to impaired synthesis, decreased affinity of eNOS for its cofactor, or increased catabolism. Biosynthesis of BH4 occurs either via a de novo pathway in which the enzyme GTP cyclohydrolase I is the rate limiting step, or via a so-called salvage pathway that utilises sepiapterin as an intermediate step. Rapid depletion of $\mathrm{BH} 4$ in the vessel wall following pharmacological inhibition of GTP cyclohydrolase I suggests that $\mathrm{BH} 4$ turnover in the endothelium is relatively high. Both depletion of GTP as well as down regulation of the expression of GTP cyclohydrolase I have been postulated to contribute to the reduced $\mathrm{BH} 4$ bioavailability in endothelial dysfunction. Recently, BH4 was shown to be a major target for oxidation by peroxynitrite suggesting that enhanced BH4 catabolism caused by prolonged oxidative stress may be an important underlying reason for the decreased BH4 bioavailability in endothelial dysfunction. ${ }^{10}$ Indeed, in hypertensive vessels BH4 oxidation could be demonstrated, leading to

Abbreviations: $\mathrm{BH} 4$, tetrahydrobiopterin; eNOS, endothelial nitric oxide synthase; FAD, flavin adenine dinucleotide; FMN, flavin mononucleotide; NADPH, nicotinamide adenine dinucleotide phosphate; NO, nitric oxide 
eNOS uncoupling with reduced formation of $\mathrm{NO}$ and increased superoxide production. ${ }^{11}$ It was hypothesised that the ratio between reduced and oxidised BH4 metabolites tightly controls superoxide formation from eNOS. ${ }^{12}$ Consistently, the antioxidant vitamin $\mathrm{C}$ has been shown to potentiate eNOS enzymatic activity by protecting $\mathrm{BH} 4$ from oxidation through its chemical stabilisation. ${ }^{13}$

\section{IMPROVING ENDOTHELIAL FUNCTION}

The above data indicate that reduced $\mathrm{BH} 4$ bioavailability may play a key role in the aetiology of endothelial dysfunction associated with conditions such as hypercholesterolaemia, hypertension, diabetes, and atherosclerosis. Administration of $\mathrm{BH} 4$ or manipulating vascular $\mathrm{BH} 4$ status by either enhancing $\mathrm{BH} 4$ synthesis or preventing its oxidation may be promising strategies for improving endothelial function and reducing cardiovascular risk. Thus far, several clinical trials have shown beneficial effects of short term BH4 supplementation on endothelial function. However, its potential as long term clinical strategy is questionable. BH4 in its active, reduced form is highly unstable and therefore not suitable for oral administration. Furthermore, administration of $\mathrm{BH} 4$ in the presence of conditions with increased oxidative stress may lead to rapid oxidative degradation of BH4 and thus limited duration of the beneficial effects. Supplementation of vitamin $\mathrm{C}$, which has been shown to prevent oxidation of $\mathrm{BH} 4$, or a combination of vitamin $\mathrm{C}$ and BH4 may prove more useful therapeutic strategies. Indeed, long term vitamin $\mathrm{C}$ administration in vivo has been shown exert a BH4 dependent stimulatory effect on NO mediated endothelial function. ${ }^{14}$ Recently, we have demonstrated that folate is also capable of BH4 dependent potentiation of eNOS function in vitro and improving NO mediated endothelial function in vivo. The exact mechanisms are not clear but may involve $\mathrm{BH} 4$ stabilisation, stimulation of $\mathrm{BH} 4$ regeneration from the inactive oxidised form, enhanced binding of $\mathrm{BH} 4$ to eNOS, or interaction with the active site of eNOS mimicking BH4. ${ }^{15}$ Interestingly, it was recently reported that statins, in addition to augmenting eNOS expression, may also potentiate GTP cyclohydrolase I gene expression and BH4 synthesis, thereby improving eNOS function. ${ }^{16}$

\section{CONCLUSION}

Normal endothelial NO synthase function is of fundamental importance for vascular homeostasis. In many vascular diseases "eNOS uncoupling" appears to be present, leading to increased superoxide and reduced NO production. The essential eNOS cofactor BH4 has a crucial role in maintaining eNOS in the optimal "coupled" state. In various vascular disease conditions loss of BH4 bioavailability and subsequent eNOS uncoupling may contribute to impaired NO mediated endothelial function. Mechanisms that modulate BH4 status in human vascular disease represent promising targets for therapeutic interventions aimed at prevention of atherosclerotic disease.

\section{Authors' affiliations}

M C Verhaar, P E Westerweel, Department of Vascular Medicine, University Medical Center Utrecht, the Netherlands

A J van Zonneveld, T J Rabelink, Department of Nephrology, Leiden University Medical Center, Leiden, the Netherlands

Correspondence to: Marianne C Verhaar, MD, PhD, Department of Vascular Medicine, F02.126, University Medical Center Utrecht, Heidelberglaan 100, 3584 CX Utrecht, The Netherlands; m.c.verhaar@azu.nl

\section{REFERENCES}

1 Schachinger V, Britten MB, Zeiher AM. Prognostic impact of coronary vasodilator dysfunction on adverse long-term outcome of coronary heart disease. Circulation 2000;101:1899-906.

2 Cai H, Harrison DG. Endothelial dysfunction in cardiovascular diseases: the role of oxidant stress. Circ Res 2000;87:840-4.

3 Xia Y, Tsai AL, Berka V, et al. Superoxide generation from endothelial nitricoxide synthase. A Ca2+/calmodulin-dependent and tetrahydrobiopterin regulatory process. J Biol Chem 1998;273:25804-8.

4 Shi W, Wang X, Shih DM, et al. Paradoxical reduction of fatty streak formation in mice lacking endothelial nitric oxide synthase. Circulation 2002; 105:2078-82.

5 Ozaki M, Kawashima S, Yamashita T, et al. Overexpression of endothelial nitric oxide synthase accelerates atherosclerotic lesion formation in apoEdeficient mice. J Clin Invest 2002;1 10:331-40.

6 Wever RMF, van Dam T, van Rijn HJM, et al. Tetrahydrobiopterin regulates superoxide and nitric oxide generation by recombinant endothelial nitric oxide synthase. Biochem Biophys Res Commun 1997;237:340-4.

7 Cosentino F, Katusic ZS. Tetrahydrobiopterin and dysfunction of endothelial nitric oxide synthase in coronary arteries. Circulation 1995;91:139-44.

8 Stroes E, Kastelein J, Cosentino F, et al. Tetrahydrobiopterin restores endothelial function in hypercholesterolemia. J Clin Invest 1997;99:41-6.

9 Heitzer T, Brockhoff C, Mayer B, et al. Tetrahydrobiopterin improves endothelium-dependent vasodilation in chronic smokers: evidence for a dysfunctional nitric oxide synthase. Circ Res 2000;86:E36-41.

10 Kuzkaya N, Weissmann N, Harrison DG, et al. Interactions of peroxynitrite, tetrahydrobiopterin, ascorbic acid, and thiols: implications for uncoupling endothelial nitric-oxide synthase. J Biol Chem 2003;278:22546-54.

11 Landmesser U, Dikalov S, Price SR, et al. Oxidation of tetrahydrobiopterin leads to uncoupling of endothelial cell nitric oxide synthase in hypertension. J Clin Invest 2003;111:1201-9.

12 Vasquez-Vivar J, Martasek P, Whitsett J, et al. The ratio between tetrahydrobiopterin and oxidized tetrahydrobiopterin analogues controls superoxide release from endothelial nitric oxide synthase: an EPR spin trapping study. Biochem J 2002;362:733-9.

13 Heller R, Unbehaun A, Schellenberg B, et al. L-ascorbic acid potentiates endothelial nitric oxide synthesis via a chemical stabilization of tetrahydrobiopterin. J Biol Chem 2001;276:40-7.

14 D'Uscio LV, Milstien S, Richardson D, et al. Long-term vitamin C treatment increases vascular tetrahydrobiopterin levels and nitric oxide synthase activity. Circ Res 2003:92:88-95.

15 Verhaar MC, Stroes E, Rabelink TJ. Folates and cardiovascular disease. Arterioscler Thromb Vasc Biol 2002;22:6-13.

16 Hattori Y, Nakanishi N, Akimoto K, et al. HMG-CoA reductase inhibitor increases GTP cyclohydrolase I mRNA and tetrahydrobiopterin in vascular endothelial cells. Arterioscler Thromb Vasc Biol 2003;23:176-82. 